\title{
Biodegradation of diuron in artificially contaminated water and seawater by wood colonized with the white-rot fungus Trametes versicolor
}

\author{
Toshio Mori ${ }^{1}$ Shiho Sudo ${ }^{1}$ Hirokazu Kawagishi ${ }^{1,2,3} \cdot$ Hirofumi Hirai $^{1,3}$
}

Received: 7 March 2018 / Accepted: 14 June 2018 / Published online: 27 June 2018

(๑) The Japan Wood Research Society 2018

\begin{abstract}
We investigated the potential of white-rot fungi for bioremediation of aqueous environments contaminated with diuron (3-(3,4-dichlorophenyl)-1,1-dimethylurea). First, diuron degradation activities of several white-rot fungi (Ceriporia lacerata, Phanerochaete chrysosporium, Phanerochaete sordida, Trametes versicolor) and a brown-rot fungus (Gloeophyllum trabeum) were evaluated under low- or high-nitrogen conditions. While G. trabeum showed hardly any degradation activity, white-rot fungi except for $C$. lacerata showed degradation activity, at least under some conditions. In particular, the activity of $T$. versicolor was high regardless of culture conditions (30-35\% degradation at both levels of nitrogen). T. versicolor degraded diuron to two metabolites, 1-(3,4-dichlorophenyl)-3-methylurea and 1-(3,4-dichlorophenyl)urea, and did not accumulate the highly toxic metabolite 3,4-dichloroaniline. Moreover, the diuron content of artificially contaminated water dramatically decreased from 1.0 to $0.012 \mu \mathrm{M}$ and artificially contaminated seawater (3.4\% sea salt, w/v) decreased to $0.405 \mu \mathrm{M}$ after incubation for 2 weeks with shaking on $T$. versicolor-colonized wood medium. These results indicated that wood medium colonized with white-rot fungi may be applicable for bioremediation and detoxification of diuron-contaminated aqueous environments.
\end{abstract}

Keywords Diuron · Bioremediation $\cdot$ Trametes versicolor $\cdot$ Colonized wood medium $\cdot$ Contaminated water

\section{Introduction}

Diuron (3-(3,4-dichlorophenyl)-1,1-dimethylurea) is a nonhormonal-type phenylurea class herbicide. Diuron binds to photosynthetic membranes, blocking electron transfer, which is needed for plant growth. Diuron has a long dissipation half-life in soils (90 days), high persistence during soil degradation and a long half-life during aqueous photolysis [1]. Therefore, diuron is widely utilized for weed control in croplands and urban areas, and is used as an antifouling paint biocide by the shipping industry. However, diuron moves

Hirofumi Hirai

hirai.hirofumi@shizuoka.ac.jp

1 Faculty of Agriculture, Shizuoka University, 836 Ohya, Suruga-ku, Shizuoka 422-8529, Japan

2 Research Institute of Green Science and Technology, Shizuoka University, 836 Ohya, Suruga-ku, Shizuoka 422-8529, Japan

3 Graduate School of Science and Technology, Shizuoka University, 836 Ohya, Suruga-ku, Shizuoka 422-8529, Japan into groundwater and seawater via runoff due to its persistence and physical characteristics [2]. Diuron contamination has been detected in rivers and seawater, where the diuron concentration exceeds the levels of European Union guidelines at some sites and in some seasons [3, 4].

Giacomazzi and Cochet [2] have summarized the toxic effects of diuron. Diuron shows low toxicity for mammals, with slight or moderate toxicity to aquatic invertebrates and fish. However, 3,4-dichloroaniline (3,4-DCA), one of diuron's main degradation metabolites, is a much more toxic compound than diuron. 3,4-DCA shows toxic and genotoxic effects on aquatic animals, as well as protozoans and bacteria. Therefore, in aiming for diuron bioremediation, it is necessary to employ microorganisms that never accumulate the ecotoxic 3,4-DCA.

Giacomazzi and Cochet [2] also reviewed diuron biodegradation. Several bacteria partially degrade diuron to form 3,4-DCA via $N$-demethylation followed by amide bond hydrolysis, and some bacteria are capable of degrading or mineralizing diuron without accumulation of 3,4DCA. Filamentous fungi also degrade diuron to form some of the same metabolites as bacteria, and accumulate 
3,4-DCA [5]. However, fungal-bacterial consortia show more efficient mineralization of diuron and lower accumulation of metabolites than the individual microorganisms [6]. Degradation of diuron by several other white-rot as well as brown-rot fungi has been investigated; however, not all the metabolites and metabolic pathways are known [7, 8]. In the degradation of diuron by wood-rot fungi, Phanerochaete chrysosporium is the best investigated fungus. Coelho-Moreira et al. [9] reported that the fungus degrades diuron to 1-(3,4-dichlorophenyl)-3-methylurea (DCPMU) and 1-(3,4-Dichlorophenyl)urea (DCPU), and that this $\mathrm{N}$-demethylation reaction is significantly inhibited by the addition of a cytochrome P450 (CYP) inhibitor. Although no 3,4-DCA was detected as a metabolite, it was presumed that the urea bond of DCPU is cleaved to form 3,4-DCA, because DCPMU and DCPU are also detected as metabolites of fungi producing 3,4-DCA from diuron [2]. P. chrysosporium is also reportedly able to form some metabolic products during mineralization of 3,4-DCA [10]. Recently, it has been reported that the white-rot fungus Ganoderma lucidum can degrade diuron via the same pathway without 3,4-DCA accumulation, and that both extracellular ligninolytic and CYP enzymes degrade diuron [11]. These observations suggest that white-rot fungi degrade diuron in collaboration with extracellular and intracellular enzymes, and do not accumulate toxic metabolites. The proposed diuron metabolic pathway of white-rot fungi is shown in Fig. 1.

Based on these observations, we considered that whiterot fungi might be useful for bioremediation of diuron-contaminated environments. Therefore, we screened white-rot fungi that are superior for diuron degradation, and determined the metabolic pathway used. Fungal bioremediation of artificially diuron-contaminated water or seawater was then demonstrated, suggesting the possible application of wood-rot fungi for remediation of diuron-contaminated aqueous environments.

\section{Materials and methods}

\section{Fungal strains and chemicals}

The brown-rot fungus Gloeophyllum trabeum KU-41 (NBRC 111644), the white-rot fungi P. chrysosporium
ME-446 (ATCC 34541), Phanerochaete sordida YK-624 (ATCC 90872), and new isolates Ceriporia lacerata K-70 and Trametes versicolor $\mathrm{K}-41$, which were isolated from natural decayed wood samples obtained from a mixed forest in Shizuoka, Japan, were used. These isolates were identified based on their 18S rRNA gene ITS and sequences (accession numbers: LC312412 and LC312413 for C. lacerate K-70; LC312414 and LC312415 for T. versicolor K-41).

Diuron was purchased from Tokyo Chemical Industry Co., Ltd., Japan. DCPU and 3,4-DCA were purchased from Wako Pure Chemical Industries, Ltd., Japan. All reagents had $>98 \%$ purity, and were used without further purification. Atrazine (Tokyo Chemical Industry) was used as an internal standard.

\section{Liquid cultivation and diuron degradation}

A disc ( $8.5 \mathrm{~mm}$ diameter) from the growing edge of mycelia on potato dextrose agar (PDA) was put into a 50-ml glass vial containing $5 \mathrm{ml}$ nitrogen-limited (LN) or high-nitrogen (HN) basal III medium (containing $1 \%$ glucose, $20 \mathrm{mM}$ 2,2-dimethylsuccinate and 1.2 or $12 \mathrm{mM}$ ammonium tartrate, respectively, $\mathrm{pH} 4.5$ ) [12], and preincubated for 5 days at $30{ }^{\circ} \mathrm{C}$ under aerobic conditions. After preincubation, $50 \mu \mathrm{l}$ of $10 \mathrm{mM}$ diuron or 3,4-DCA (DMSO solution) was added to each culture, then the vial was sealed with a butyl rubber stopper and aluminum seal. After a further 7 days of incubation, the culture was homogenized following the addition of methanol $(5 \mathrm{ml})$ and internal standard (atrazine). The obtained homogenate was centrifuged $(2500 \times g$ for $10 \mathrm{~min})$ and the resulting supernatant was filtered (Millex LG filter, pore size $0.20 \mu \mathrm{m}$, Merck Millipore Ltd., Ireland). Then the concentrations of diuron and its metabolites were measured by high-performance liquid chromatography (HPLC). The HPLC system (JASCO PU-2089 pump with a JASCO MD-2018 PDA detector) was equipped with an Inertsil ODS-3 column $(4.6 \times 250 \mathrm{~mm})$ and eluted with $50 \%$ aqueous methanol. The maximum absorbance of each compound (atrazine at $220 \mathrm{~nm}$, diuron at $248 \mathrm{~nm}$, DCPU at $210 \mathrm{~nm}$, and 3,4-DCA at $208 \mathrm{~nm}$ ) were used for quantification. As necessary, the supernatant was evaporated to dryness, followed by dissolving it in methanol $(0.5 \mathrm{ml})$, and then analyzing it by HPLC.

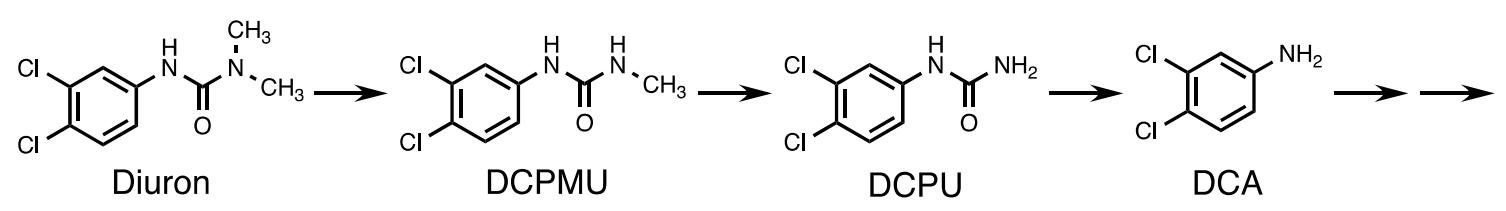

Fig. 1 Proposed Diuron metabolic pathway of white rot fungi. Diuron 3-(3,4-dichlorophenyl)-1,1-dimethylurea, DCPMU 1-(3,4-dichlorophenyl)-3-methylurea, DCPU 1-(3,4-dichlorophenyl) urea, DCA 3,4-dichloroaniline 
To determine the effect of salt at seawater concentration on diuron degradation, $T$. versicolor $\mathrm{K}-41$ was preincubated in $5 \mathrm{ml} \mathrm{HN}$ medium containing 0 or $3.4 \%$ sea salt (SigmaAldrich). Diuron was added to the culture in the same manner as described above, and the culture was incubated further.

\section{Enzyme assay}

The culture supernatants on day 5 were obtained from $\mathrm{LN}$ and HN cultures. The supernatants were used for assays of ligninolytic enzyme activities. Manganese peroxidase (MnP), lignin peroxidase (LiP) and laccase activities were measured as described previously [13]. Activities were calculated from absorbance change at $469 \mathrm{~nm}$ (for $\mathrm{MnP}$ and laccase, 2,6-dimethoxyphenol oxidation, $\varepsilon_{469}=49600 \mathrm{M}^{-1} \mathrm{~cm}^{-1}$ ) and $310 \mathrm{~nm}$ (for LiP, oxidation of veratryl alcohol, $\varepsilon_{310}=9300 \mathrm{M}^{-1} \mathrm{~cm}^{-1}$ ) [14].

\section{Metabolite identification}

Metabolites were obtained from 14-day cultures (140 vials) of $T$. versicolor $\mathrm{K}-41$ incubated on $\mathrm{HN}$ medium. Filtrate was separated from the culture homogenate prepared as described above, and was extracted with ethyl acetate. In this case, the internal standard was not added to the culture, and quantification was not performed. A concentrated ethyl acetate fraction was fractionated by silica gel column chromatography (silica $60 \mathrm{~N}, 800 \mathrm{~g}, \varphi 60 \times 500 \mathrm{~mm}$ ) eluted with $n$-hexane, ethyl acetate, and methanol [hexane/EtOAc/ $\mathrm{MeOH}=6 / 4 / 0,5 / 5 / 0,4 / 6 / 0,3 / 7 / 0,2 / 8 / 0,1 / 9 / 0,0 / 10 / 0$, $0 / 9 / 1,0 / 8 / 2$ (1 1 each) and then $0 / 0 / 10$ (3 1)]. Fractions were collected every $100 \mathrm{ml}$. Fractions containing metabolites (fractions 62-69 for product I, fractions 85-100 for product II) were further purified by HPLC on a Develosil C30-UG-5 column $(4.5 \times 250 \mathrm{~mm}$; Nomura Chemical Co., Ltd., Japan) using $40 \%$ aqueous methanol. Electrospray ionization (ESI) mass spectrometry and ${ }^{1} \mathrm{H}$-nuclear magnetic resonance (NMR) spectrometry were used the same equipment as previously described [15]. Diuron metabolites were ESI-mass analyzed in negative ionization mode, and were dissolved in $\mathrm{CD}_{3} \mathrm{OD}$ for NMR analysis.

\section{Fungal cultivation on wood substrate, and diuron removal from artificially contaminated water}

A mycelial plate (PDA, $9 \mathrm{~cm}$ i.d.) was homogenized with $25 \mathrm{ml}$ distilled water. Homogenate and additional distilled water were mixed well with wood substrate (180 $\mathrm{g}$ beech wood meal and $20 \mathrm{~g}$ wheat bran meal) to meet a solid content of $28 \%$. The wood substrate was incubated for 2 weeks at $30{ }^{\circ} \mathrm{C}$ with $T$. versicolor $\mathrm{K}-41$ to allow colonization.
Artificial contaminated water (containing $10 \mathrm{mM} \mathrm{CaCl}_{2}$ to prevent pesticide sorption as described by $\mathrm{Li}$ et al. [16]) and artificial contaminated seawater (containing 3.4\% (w/v) sea salt), both artificially contaminated with $1 \mu \mathrm{M}$ diuron, were prepared. $T$. versicolor K-41-colonized wood medium was added to $20 \mathrm{ml}$ of artificially contaminated water and seawater at $5 \%($ dry $w / v)$. The suspension was rotated at $120 \mathrm{rpm}$ at $30{ }^{\circ} \mathrm{C}$ for 2 weeks under ambient atmosphere. At the end of the reaction, the suspension was centrifuged at $8000 \times g$ for $10 \mathrm{~min}$ to separate supernatant and a precipitate. After the addition of internal standard, the supernatant was evaporated, then extracted with ethyl acetate to remove hydrophilic impurities. The extract was dried and redissolved in methanol. The precipitate was extracted twice with methanol $(20 \mathrm{ml})$. The methanol-soluble fraction was purified and redissolved in methanol in the same manner as the supernatant. Recovery of diuron was analyzed by HPLC. Wood substrate without any inoculum (abiotic) and autoclaved colonized wood medium (sterilized) were used as controls.

\section{Results and discussion}

\section{Selection of diuron-degrading wood-rot fungi and their metabolites}

To develop a bioremediation method for aqueous contamination with diuron, we screened for wood-rot fungi that show higher diuron degradation activity and do not accumulate toxic compounds such as 3,4-DCA. Fig. 2 shows diuron degradation by wood-rot fungi after a 7- and 14-day incubation period in LN and $\mathrm{HN}$ media. Generally, increasing nitrogen concentration in liquid culture promotes growth of white-rot fungi. In contrast, the ligninolytic activity of some white-rot fungi is inhibited under $\mathrm{HN}$ conditions, and secondary metabolism, including ligninolytic activity, is stimulated by nitrogen limitation [17]. G. trabeum, used as a representative brown-rot fungus, slightly degraded diuron in $\mathrm{HN}$ medium, but not in LN medium. On the other hand, the white-rot fungus $P$. chrysosporium had degraded almost $20 \%$ of the diuron in both media at 7 days, and $P$. sordida showed diuron-degrading activity only in LN medium. In diuron metabolism by $P$. chrysosporium and G. lucidum, the following facts have been shown; (1) diuron degradation was correlated with ligninolytic activity on nitrogen-limited and wood culture, (2) laccase was able to degrade diuron, and (3) diuron metabolism was considerably inhibited by addition of CYP inhibitors [9, 11, 18]. These facts indicate that ligninolytic enzymes are not mandatory to diuron degradation of white rot fungi, and also suggest that white rot fungi can degrade diuron under the condition in which the CYP isozyme involved in diuron metabolism is expressed. 
Fig. 2 Diuron recovery from fungal cultures grown on $\mathrm{LN}$ and $\mathrm{HN}$ media after 0,7 and 14 days of incubation (black, gray and white bar, respectively). Values are means \pm standard deviation of triplicate samples

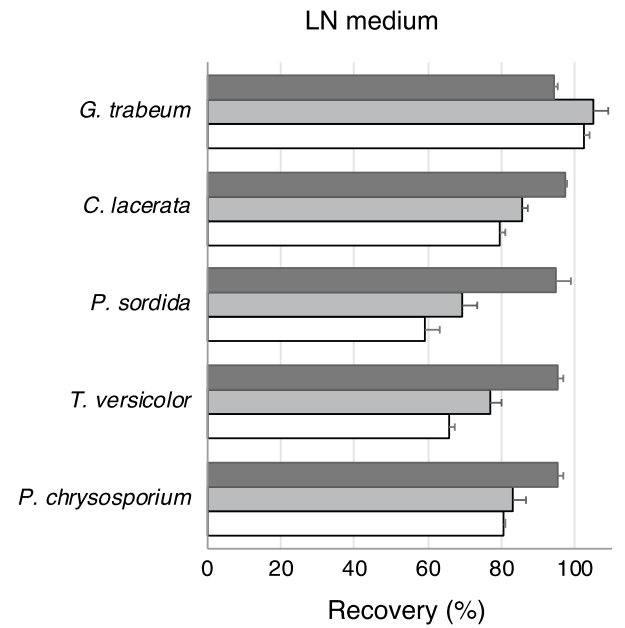

Therefore, as shown in Fig. 2, there is considered that diuron degradation of white rot fungi was not always influenced by nitrogen concentration in the culture medium. There are some reports describing the degradation of neonicotinoid herbicides by $P$. sordida, and the metabolites are presumably formed via $N$-demethylation or $N$-nitro reduction catalyzed by CYPs $[15,19]$. However, no metabolites were detected in single cultures of these two Phanerochaete species based on HPLC in the present study. T. versicolor K-41 was one of the best fungi for diuron degradation, and showed higher diuron degradation in $\mathrm{HN}$ than $\mathrm{LN}$ medium after a 7-day incubation period (Fig. 2). Bending and co-authors [7] reported diuron degradation by a wide range of wood-rot fungi including $T$. versicolor, but did not report any correlation between ligninolytic activity and diuron degradation. Thus, there is still a lack of information on metabolites and the mechanisms of diuron degradation by T. versicolor. Leatham and Kirk [17] described strong stimulation of ligninolytic activity of $T$. versicolor under nitrogen-limited conditions and inhibition of activity by high nitrogen concentration, though moderate ligninolytic activity remained. In this study, T. versicolor $\mathrm{K}-41$ was showed $\mathrm{MnP}$ and laccase activity under $\mathrm{HN}$ condition $(1.5 \pm 0.6 \mathrm{U} / 1$ and $3.2 \pm 0.2 \mathrm{U} / 1$, respectively), while the higher activities were presented under $\mathrm{LN}$ condition (2.4 U/1 $\mathrm{MnP}$ and $5.2 \pm 0.1 \mathrm{U} / \mathrm{l}$ laccase). Trace levels of LiP activity were detected from both cultures. Nevertheless, diuron degradation activities under both nitrogen condition were showed almost same level. Therefore, it was presumed that T. versicolor $\mathrm{K}-41$ is able to degrade diuron by ligninolytic extracellular and non-ligninolytic intracellular enzymes, as described above for other white-rot fungi. T. versicolor K-41 showed higher diuron degradation activity regardless to nitrogen concentration, the degradation rates of $T$. versicolor $\mathrm{K}-41$ were equal $P$. sordida under LN condition and $P$. chrysosporium under $\mathrm{HN}$ condition. Although mycelial growth is expected to vary depending on culture condition, it is important to show the high degradation activity regardless of condition to apply for bioremediation use. Therefore, $T$. versicolor $\mathrm{K}-41$ was used for subsequent experiments.

A peak (product I, $17.70 \mathrm{~min}$ ) having a UV spectrum resembling diuron was detected in an ethyl acetate extract of culture homogenate of T. versicolor K-41 after HPLC, as shown in Fig. 3. Product I showed a molecular ion peak at $\mathrm{m} / \mathrm{z} 203[\mathrm{M}-\mathrm{H}]^{-}$and resulting chlorine substitution peaks at $\mathrm{m} / \mathrm{z} 205$ and 207. A fragment ion peak at $\mathrm{m} / \mathrm{z}, 160$ $\left[\mathrm{M}-\mathrm{CONH}_{2}\right]^{-}$and chlorine substitution peaks at $\mathrm{m} / \mathrm{z} 162$ and 164 were observed. From these results and the ${ }^{1} \mathrm{H}-\mathrm{NMR}$ spectrum, product I was estimated as DCPU, and was finally identified by comparison with authentic standard. In addition to product I, product II $(24.0 \mathrm{~min})$ was detected as a minor peak on HPLC in a 50-100 times concentrated extract without internal standard. Because product II presented almost

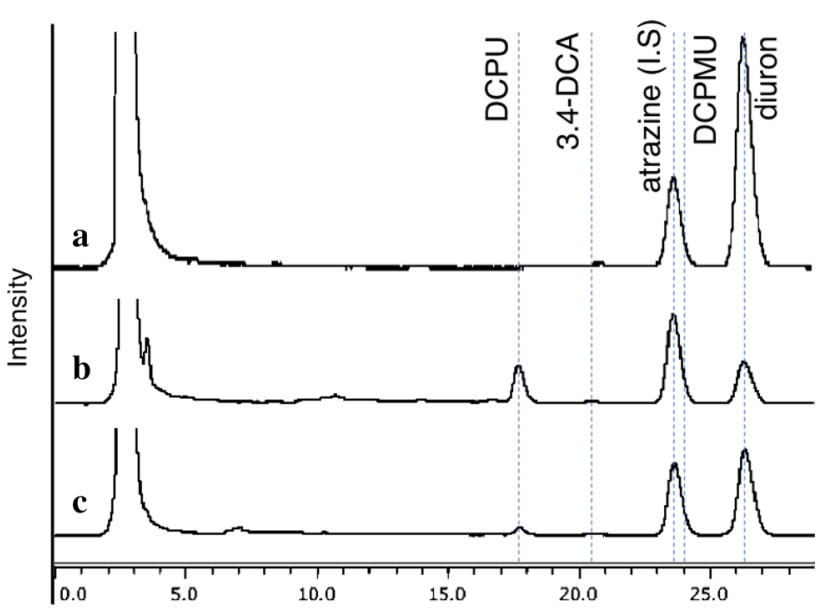

Fig. 3 High-performance liquid chromatograms (210 nm, maximum absorption wavelength of DCPU) of concentrated extracts obtained from whole $\mathrm{HN}$ cultures of $T$. versicolor $\mathrm{K}-41$ that were incubated with diuron. a, b Show chromatograms of extracts from nonsaline cultures incubated with diuron for an additional 0 and 14 days, respectively. c Shows a chromatogram of extracts from hypersaline cultures incubated with diuron for an additional 14 days 
the same retention time as the internal standard (atrazine, $23.6 \mathrm{~min}$ ), it could not be detected in quantification experiments with internal standard by HPLC (Fig. 3). Product II showed a relatively weak molecular ion peak at $\mathrm{m} / z 217$ $[\mathrm{M}-\mathrm{H}]^{-}$(and two ion peaks indicating chlorine substitution), and a fragment ion peak at $\mathrm{m} / z, 160\left[\mathrm{M}-\mathrm{CONHCH}_{3}\right]^{-}$was observed. Its ${ }^{1} \mathrm{H}-\mathrm{NMR}$ spectrum was identical to one previously reported [20], and therefore, product II was identified as DCPMU. On the other hand, although 3,4-DCA was not detectable by HPLC of unconcentrated supernatant, a slight amount $(2.3 \pm 0.2 \mu \mathrm{M})$ was detected in concentrated extract prepared from a 14-day culture (Fig. 3). Therefore, to reveal whether 3,4-DCA is accumulated or is a transient metabolite, a degradation experiment for 3,4-DCA was performed. In that experiment, the concentration of 3,4-DCA decreased to below the lower detection limit after a 2-day incubation, which indicated that 3,4-DCA is just one of the intermediates produced during diuron metabolism of T. versicolor $\mathrm{K}-41$, and that the fungus is able to metabolize 3,4-DCA quickly under these experimental conditions, the same as $P$. chrysosporium [10].

\section{Diuron removal from artificially contaminated water}

Because diuron is typically present in river and seawater [3, 4], diuron degradation by $T$. versicolor $\mathrm{K}-41$ under hypersaline conditions was investigated. The recovery of diuron and DCPU from normal and hypersaline (3.4\% sea salt) cultures of T. versicolor K-41 is shown in Fig. 4. Diuron degradation and DCPU production by $T$. versicolor were inhibited by the addition of sea salt. DCPU yields from nonsaline and hypersaline cultures incubated with diuron for an additional 14 days were $10.8 \pm 4.1$ and $2.0 \pm 0.4 \mu \mathrm{M}$,

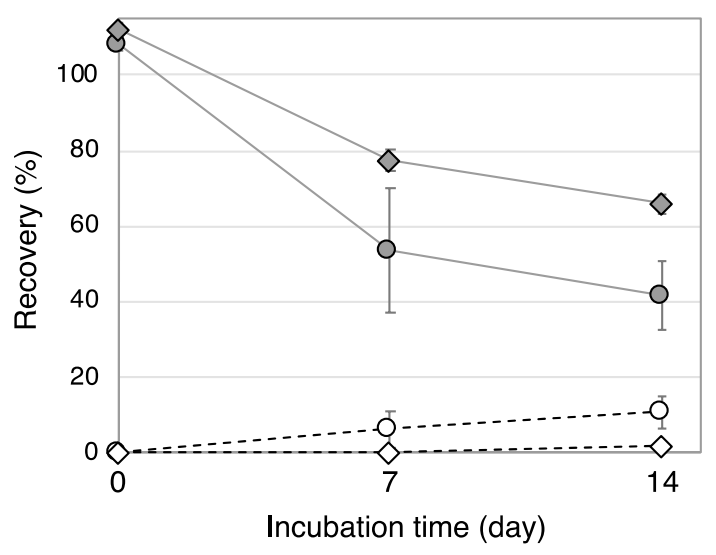

Fig. 4 Time courses of diuron degradation and DCPU accumulation by $T$. versicolor $\mathrm{K}-41$ under hypersaline and nonsaline conditions. The filled and open symbols indicated diuron degradation and DCPU accumulation under hypersaline (diamonds) and nonsaline (circles) conditions, respectively. Values are means \pm standard deviation of triplicate samples respectively. And respective $2.3 \pm 0.2$ and $1.8 \pm 0.1 \mu \mathrm{M}$ of 3,4 -DCA were recovered from the cultures. No DCPMU was detectable from extracts of single cultures. However, diuron degradation in hypersaline conditions was $60 \%$ of that under nonsaline conditions. Total recoveries of diuron and its metabolites were $55 \%$ from nonsaline culture and $70 \%$ from hypersaline culture. The phenomenon that the total recovery rate of diuron and its metabolites decreases by fungus treatment has been also observed in P. chrysosporium and G. lucidum [9, 11].

Next, to estimate the remediation potential of T. versicolor $\mathrm{K}-41$ in a diuron-contaminated aqueous environment, the efficiency of diuron removal from artificially diuroncontaminated water and seawater was evaluated. In 2007, European-wide environmental quality standards for 33 high-priority hazardous substances including diuron were published [21]. Annual average and maximum allowable concentrations of diuron for surface water have been set at 0.2 and $1.8 \mu \mathrm{g} / \mathrm{L}$, respectively. However, because of experimental limitations to quantitation, we chose a diuron concentration in artificially contaminated water of $1 \mu \mathrm{M}$, a much higher concentration than allowable under these standards.

Because these fungi need utilizable saccharides for survival, we used wood as a source of nutrients and as an immobilized carrier for the fungi for bioremediation of aqueous contaminants. Wood colonized with T. versicolor K-41 was suspended in water or seawater artificially contaminated with diuron to evaluate the adsorption and co-metabolism of diuron relative to uninoculated wood substrate and sterilized controls. As shown in Fig. 5, calculated recovery

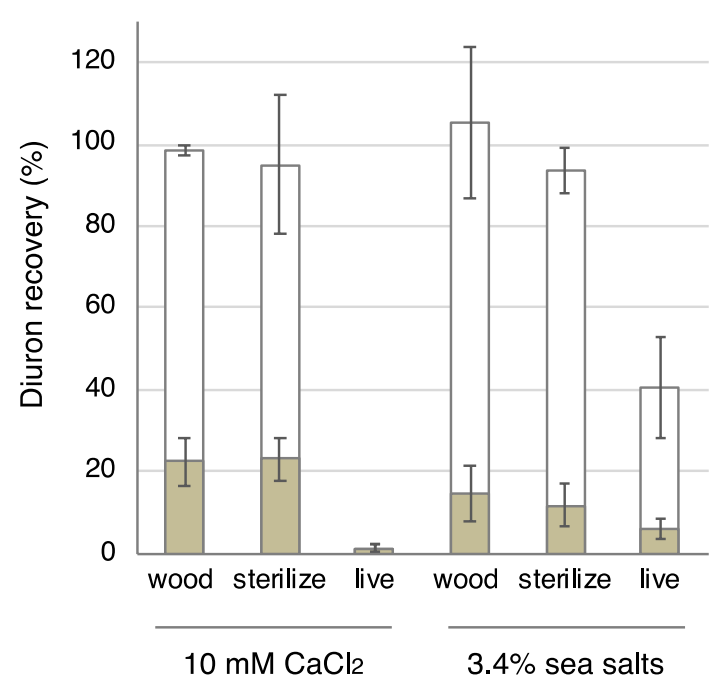

Fig. 5 Recovery of diuron from artificially contaminated water (containing $10 \mathrm{mM} \mathrm{CaCl}_{2}$ ) and seawater (containing 3.4\% sea salts) incubated for 2 weeks with a wood substrate (abiotic), and sterilized and unsterilized (live) wood medium colonized with $T$. versicolor K-41. Each column indicates total diuron recovery, and the gray portion represents the aqueous-soluble portion 
rates of diuron from abiotic and sterilized controls were $93.7-105.5 \%$ of the initial amount $(1 \mu \mathrm{M})$. In these experiments, $20 \%$ or less of the diuron was recovered from the aqueous phase of controls; the other $80 \%$ was absorbed to the wood substrate. Additionally, it seemed that sterilized mycelium was not involved in diuron adsorption, but that sea salt slightly affected diuron sorption to woody materials. Wood adsorption of aromatic pesticides having a structure similar to diuron has been reported [22]. We speculated that some components of sea salts affected diuron adsorption to the woody materials, because it has been reported that adsorption of diuron to soil is greatly affected by the salt type and concentration [16].

During treatment of artificially contaminated water with T. versicolor K-41-colonized wood medium, diuron was almost completely degraded (98.7\%) during a 2-week incubation period (Fig. 5). The fungus might have been able to degrade diuron effectively due to adsorption of diuron to the medium. In artificially contaminated seawater, on the other hand, the degradation rate of diuron was lower than in contaminated water without saline, as observed in liquid cultures. However, more than half of the diuron was degraded by the T. versicolor K-41 colonizing the wood medium. No metabolites were confirmed by HPLC after fungal treatment of the contaminated water and seawater. The absence of metabolite detection was presumably due to the low initial amount of diuron, so that the amount of metabolites did not reach the level detectable by HPLC. These results suggested that $T$. versicolor K-41-colonized wood medium can be utilized for bioremediation of diuron-contaminated aqueous environments, especially under salt-free conditions.

Several diuron degrading filamentous fungi have been isolated from environments such as diuron-treated fields, and they are also able to degrade the related phenyl urea herbicides [23]. Some reports have also described filamentous fungi that are capable of growth in soil contaminated with diuron and may be useful for bioremediation of diuron-contaminated soil [24, 25]. On the other hand, a wide range of wood-rot fungi, including the edible mushroom Pleurotus ostreatus, have the capability to degrade diuron and related herbicides $[7,8]$. Nevertheless, the growth of wood-rot fungi is usually inhibited when introduced into a soil environment, which is different from wood in many respects due to the low amount of available nutrients and competitive indigenous microorganisms [26]. However, utilization of lignocellulosic material such as wood allows growth of white-rot fungi in soil environments. Actually, there are several reports on successful soil remediation by applying lignocellulosic materials and white-rot fungi [26]. These observations suggest that white-rot fungi may be applicable for bioremediation in several environments by establishing conditions appropriate for their growth. In the present study, diuron degradation in artificially contaminated water was demonstrated by the white-rot fungus $T$. versicolor $\mathrm{K}-41$ after it had colonized wood meal, which was utilized as both a source of nutrients and an immobilizing carrier. In natural environments, various factors are involved in biodegradation of organic pollutants; therefore, additional study is needed for evaluation of the effectiveness of fungal-colonized wood in bioremediation of actual polluted aqueous environments. Still, there is a possibility that inexpensive bioremediation methods for aqueous pollution utilizing spent mushroom wastes of edible or medicinal mushrooms (such as $P$. ostreatus and T. versicolor), which are currently discarded as industrial wastes, can be developed if suitable procedures are established by future studies.

\section{References}

1. Moncada A (2004) DRP report: environmental fate of diuron. California Department of Pesticide Regulation, USA

2. Giacomazzi S, Cochet N (2004) Environmental impact of diuron transformation: a review. Chemosphere 56:1021-1032

3. Kaonga CC, Takeda K, Sakugawa H (2015) Diuron, Irgarol 1051 and Fenitrothion contamination for a river passing through an agricultural and urban area in Higashi Hiroshima City, Japan. Sci Total Environ 518-519:450-458

4. Harino H, Eguchi S, Arai T, Ohji M, Yamamoto Y, Miyazaki N (2010) Antifouling biocides contamination in sediment of coastal waters from Japan. Coast Mar Sci 34:230-235

5. Tixier C, Sancleme M, Bonnemoy F, Cuer A, Veschambre H (2001) Degradation products of a phenylurea herbicide, diuron: synthesis, ecotoxicity, and biotransformation. Environ Toxicol Chem 20:1381-1389

6. Ellegaard-Jensen L, Knudsen BE, Johansen A, Albers CN, Aamand J, Rosendahl S (2014) Fungal-bacterial consortia increase diuron degradation in water-unsaturated systems. Sci Total Environ 466-467:699-705

7. Bending GD, Friloux M, Walker A (2002) Degradation of contrasting pesticides by white rot fungi and its relationship with ligninolytic potential. FEMS Microbiol Lett 212:59-63

8. Khadrani A, Seigle-Murandi F, Steiman R, Vroumsia T (1999) Degradation of three phenylurea herbicides (chlortoluron, isoproturon and diuron) by micromycetes isolated from soil. Chemosphere 38:3041-3050

9. Coelho-Moreira JDS, Bracht A, da Silva de Souza AC, Oliveira RF, de Sá-Nakanishi AB, de Souza CGM, Peralta RM (2013) Degradation of diuron by Phanerochaete chrysosporium: role of ligninolytic enzymes and cytochrome P450. Biomed Res Int 2013:251354. https://doi.org/10.1155/2013/251354

10. Sandermann H, Heller W, Hertkorn N, Hoque E, Pieper D, Winkler R (1998) A new intermediate in the mineralization of 3,4-dichloroaniline by the white rot fungus Phanerochaete chrysosporium. Appl Environ Microbiol 64:3305-3312

11. Coelho-Moreira J, da S, Brugnari, Sá-Nakanishi T, Castoldi AB, de Souza R, Bracht CGM, Peralta A RM (2018) Evaluation of diuron tolerance and biotransformation by the white-rot fungus Ganoderma lucidum. Fungal Biol 122:471-478

12. Tien M, Kirk TK (1988) Lignin peroxidase of Phanerochaete chrysosporium. Methods Enzymol 161:238-249 
13. Hirai H, Kondo R, Sakai K (1994) Screening of lignin-degrading fungi and their ligninolytic enzyme activities during biological bleaching of kraft pulp. Mokuzai Gakkaishi 40:980-986

14. Mester T, Field JA (1998) Characterization of a novel manganese peroxidase-lignin peroxidase hybrid isozyme produced by Bjerkandera species strain BOS55 in the absence of manganese. J Biol Chem 273:15412-15417

15. Mori T, Wang J, Tanaka Y, Nagai K, Kawagishi H, Hirai H (2017) Bioremediation of the neonicotinoid insecticide clothianidin by the white-rot fungus Phanerochaete sordida. J Hazard Mater 321:586-590

16. Li H, Teppen BJ, Laird DA, Johnston CT, Boyd SA (2006) Effects of increasing potassium chloride and calcium chloride ionic strength on pesticide sorption by potassium- and calciumsmectite. Soil Sci Soc Am J 70:1889-1895

17. Leatham GF, Kirk TK (1983) Regulation of ligninolytic activity by nutrient nitrogen in white-rot basidiomycetes. FEMS Microbiol Lett 16:65-67

18. Fratila-Apachitei LE, Hirst JA, Siebel MA, Gijzen HJ (1999) Diuron degradation by Phanerochaete chrysosporium BKM-F-1767 in synthetic and natural media. Biotechnol Lett 21:147-154

19. Wang J, Hirai H, Kawagishi H (2012) Biotransformation of acetamiprid by the white-rot fungus Phanerochaete sordida YK-624. Appl Microbiol Biotechnol 93:831-835
20. Van Boven M, Laruelle L, Daenens P (1990) HPLC analysis of diuron and metabolites in blood and urine. J Anal Toxicol 14:231-234

21. Crane M, Babut M (2007) Environmental quality standards for water framework directive priority substances: challenges and opportunities. Integr Environ Assess Manag 3:290-296

22. Rodriguez-Cruz S, Andrades MS, Sanchez-Camazano M, Sanchez-Martin MJ (2007) Relationship between the adsorption capacity of pesticides by wood residues and the properties of woods and pesticides. Environ Sci Technol 41:3613-3619

23. Sørensen SR, Bending GD, Jacobsen CS, Walker A, Aamand J (2003) Microbial degradation of isoproturon and related phenylurea herbicides in and below agricultural fields. FEMS Microbiol Ecol 45:1-11

24. Esposito E, Paulillo SM, Manfio GP (1998) Biodegradation of the herbicide diuron in soil by indigenous actinomycetes. Chemosphere 37:541-548

25. Wang Y, Li H, Feng G, Du L, Zeng D (2017) Biodegradation of diuron by an endophytic fungus Neurospora intermedia DP8-1 isolated from sugarcane and its potential for remediating diuroncontaminated soils. PLoS One 12:e0182556

26. Baldrian $\mathrm{P}$ (2008) Wood-inhabiting ligninolytic basidiomycetes in soils: ecology and constraints for applicability in bioremediation. Fungal Ecol 1:4-12 